\title{
Variability of serum high density lipoprotein cholesterol concentration in healthy subjects in a three
} year term*

\author{
KEIKO CHIBA, TAKAO WATANABE, AND MASAYUKI IKEDA \\ From the Department of Environmental Health, Tohoku University School of Medicine, Sendai, 980 Japan
}

SUMmaRY The measurements of serum high density lipoprotein cholesterol (HDL-C), total cholesterol (TC), and triglyceride (TG) were repeated in 170 apparently healthy farming women once a year for three years (1980-2) to examine the variability of the results within the same individual. Although the mean HDL-C and TC values varied to some extent year by year, higher correlation coefficients between any pair and smaller coefficients of variation in the three annual measurements indicated that the within subject variability of $\mathrm{HDL}-\mathrm{C}$ and TC is much smaller than that of TG. Variation of HDL-C appeared to be associated with lipidaemia. When the effects of age and menopause were eliminated, it was found that non-normolipidaemic people had wider individual variation both in HDL-C and TG. The importance of TG determination for HDL-C evaluation in health examination is emphasised.

In recent years measurement of serum high density lipoprotein cholesterol (HDL-C) concentration has become a favourite item of health examination in various populations, ${ }^{1-3}$ especially as a predictive indicator of ischaemic heart diseases. ${ }^{45}$ Since the predictive power of single determination of HDL-C in health examination may depend on the variability under normal life conditions, ${ }^{1}$ it is considered necessary to investigate the possible magnitude of variation within the same apparently healthy individual.

In the present study a group of 170 farming women were examined once a year for three years for their HDL-C concentrations as well as total cholesterol (TC) and triglyceride (TG) in the serum. The results will be described on the within subject variation of HDL-C and its relation with TC and TG.

\section{Methods}

POPULATION

The examinees were 170 apparently healthy farming women in a single prefecture of Miyagi in Japan who participated in each and every (three) annual health examinations in the summers of 1980-2; they had no history of serious medical care during the study

- Part of this work was presented at the 42nd annual meeting of the Japanese Society of Public Health held in Yokohama on 8-11 November 1983. period. The mean age ( $\pm \mathrm{SD})$ of the examinees in 1980 was $45 \cdot 0 \pm 9.7$ years (range 22 to 50 ).

HEALTH EXAMINATION PROCEDURE AND SERUM BIOCHEMISTRY

Examination design, blood sampling, and methods in serum biochemistry were previously described in detail. ${ }^{1}$ Serum high density lipoprotein cholesterol (HDL-C), TC, and TG values were cited from the previous study. ${ }^{1}$

STATISTICAL ANALYSES

Difference between two means were evaluated using the $t$ test.

\section{Results}

The mean $( \pm S D)$ levels of HDL-C, TC, and TG in the serum in the three year term are summarised in table 1 ; there was a slight yet significant $(p<0.01)$ decrease in the HDL-C concentration in 1981 and 1982 compared with the level in 1980 , and the TC concentration in 1981 was significantly $(p<0.01)$ lower than the concentration in the other two years.

When the variability of examination results was evaluated in term of the correlation coefficient between any pair of the three annual results - that is, $1980 \vee 1981,1980 \vee 1982$, and $1981 v 1982$-high coefficients with statistical significance $(r=0.72$, 
0.68 , and 0.70 in the order; $p<0.01$ ) were obtained in the case of $\mathrm{HDL}$, indicating that a given individual with high (or low) HDL-C concentration in the 1980 examination will probably give similarly high (or low) values in 1981 and 1982. Such was also the case with TC $(r=0.65,0.71,0.76 ; p<0.01)$, whereas somewhat weaker associations were observed when TG was examined $(r=0.49,0.58,0.55 ; \mathrm{p}<0.01)$.

Table 1 Serum HDL-C, TC, and TG concentrations in three years

\begin{tabular}{lllll}
\hline Measurement (unit) & 1980 & \multicolumn{2}{c}{1981} & \multicolumn{2}{c}{1982} \\
\hline HDL-C $(\mathrm{mg} / 100 \mathrm{ml})$ & $49 \cdot 6 \pm 9 \cdot 8^{*}$ & $46.5 \pm 10 \cdot 8$ & $46.5 \pm 9 \cdot 8$ \\
TC $\quad(\mathrm{mg} / 100 \mathrm{ml})$ & $199 \pm 37 \cdot 0$ & $184 \pm 37.4^{*}$ & $200 \pm 34.6$ \\
TG $\quad(\mathrm{mg} / 100 \mathrm{ml})$ & $110 \pm 53.5$ & $103 \pm 50 \cdot 4$ & $107 \pm 55.7$ \\
\hline
\end{tabular}

The same examinees (170 women) were followed for three years. Numbers in table are mean \pm SD.

-Difference from other values is statistically significant $(p<0.01)$.

The magnitude of variability in HDL-C on an individual basis was further examined by calculating the coefficient of variation (CV) for each individual from three measurements of HDL-C concentration in her serum on three successive occasions. When such calculation for $\mathrm{CV}(\%)$ was made for each of the 170 examinees, the results distributed normally with a mean $\pm S D$ of $10.9 \pm 6.0 \%$. A similar calculation for TC gave a mean \pm SD of $10 \cdot 1 \pm 5 \cdot 0 \%$. In the case of TC, however, the value obtained $(26.4 \pm 14.0 \%)$ was significantly larger $(p<0.01)$ than the counterpart values for HDL-C and TC, indicating that the TG concentration of the same individual may vary in a wider range depending on the examination occasion than HDL and TC.

In order to examine possible effect of lipidaemia on the extent of variation in HDL-C concentration of the same subject in the three year term the $\mathbf{1 7 0}$ examinees were classified into two subgroups depending on TC and TG concentrations-those whose TC and TG concentrations were always less than $250 \mathrm{mg} / 100 \mathrm{ml}$ and $150 \mathrm{mg} / 100 \mathrm{ml}$, respectively, throughout the three examinations (normolipidaemic group; 112 subjects) and those whose TC or TG concentrations exceeded the level at any one or more occasions in the three examinations (non-normolipidaemic group; 58 subjects). The comparison of HDL-C values between three pairs out of the three annual results-that is, $1980 v 1981$, $1980 v 1982$, and $1981 v 1982$-gave correlation coefficients of $0.70,0.67,0.73,(p<0.01)$ for the normolipidaemic group and $0.74,0.74,0.61$ $(p<0.01)$ for the non-normolipidaemic group; no statistical significance $(p>0 \cdot 10)$ could be detected between the two sets of correlation coefficients after Z-transformation. When the coefficients of variation for HDL-C, TC, and TG were compared between the normolipidaemic and non-normolipidaemic groups, however, the values for the former group were $10.0 \pm 5.6 \%, 10 \cdot 2 \pm 5.0 \%$, and $24.5 \pm 12.7 \%$ whereas they were $12 \cdot 7 \pm 6.4 \%, 9.8 \pm 5.0 \%$, and $30 \cdot 1 \pm 15 \cdot 8 \%$ for the latter. Both HLD-C and TG in the non-normolipidaemic group were significantly $(p<0.01)$ larger than the counterpart values in the normolipidaemic group, suggesting the possibility that the increase in variability of HDL-C concentration is associated with raised variability of TG concentration. The previous study showed that biological factors such as sex, age, and menopause affect TC and TG, but not HDL-C. ${ }^{1}$ Accordingly, 48 premenopausal women aged 40-59 were selected and CV(\%) of HDL-C, TC, and TG in the three year term were calculated on individual bases. The results were pooled into the normolipidaemic group $(n=31)$ and the non-normolipidaemic group $(n=17)$ depending on the TC and TG concentrations as previously described (table 2). It was evident that the mean $C V$ value for $H D L-C$ was significantly $(p<0.01-0.05)$ larger in the non-normolipidaemic group than in the normolipidaemic group (by $70 \%$ ) as was the case of TG (by 33\%), whereas essentially there was no change in TC.

\section{Discussion}

By tracing the concentrations of HDL-C, TC, and TG in the serum of 170 apparently healthy women in a three year term, the present study makes clear that HDL-C concentration is highly reproducible with

Table 2 Coefficients of variation in a three year term for serum HDL-C,TC, and TG in the premenopausal women aged 40-59

\begin{tabular}{|c|c|c|c|c|}
\hline Group & $\begin{array}{l}\text { No of } \\
\text { examinees }\end{array}$ & $\begin{array}{l}H D L \\
(\%)\end{array}$ & $\begin{array}{l}T C \\
(\%)\end{array}$ & $\begin{array}{l}T G \\
(\%)\end{array}$ \\
\hline $\begin{array}{l}\text { Total } \\
\text { Normolipidaemic } \\
\text { Non-normolipidaemic }\end{array}$ & $\begin{array}{l}48 \\
31 \\
17\end{array}$ & $\begin{aligned} 10 \cdot 4 & \pm 5 \cdot 5 \\
8 \cdot 3 & \pm 4 \cdot 6 \\
14 \cdot 1 & \pm 5 \cdot 1^{* *}\end{aligned}$ & $\begin{array}{l}10 \cdot 3 \pm 4 \cdot 0 \\
10 \cdot 1 \pm 3 \cdot 4 \\
10 \cdot 7 \pm 5 \cdot 1\end{array}$ & $\begin{array}{l}27 \cdot 6 \pm 16 \cdot 3 \\
24 \cdot 7 \pm 13 \cdot 9 \\
32 \cdot 9 \pm 19 \cdot 3^{*}\end{array}$ \\
\hline
\end{tabular}

For definition of "normolipidaemic" and "non-normolipidaemic" see text. Numbers in table are mean \pm SD of coefficient of variation, which was calculated for each individual from her three annual measurements.

*,*: Increase from value for normolipidaemic group is statistically significant $\left({ }^{*} p<0.05, * * p 0.01\right)$. 
small magnitude of variation when the same individual is repeatedly examined and that variability in the HDL-C concentration appears to be associated with a raised fluctuation in the TG concentration. It should be emphasised that all the measurements were conducted by the same person in the same laboratory using the same set of instruments and endeavouring to avoid such systemic errors as inter laboratory difference even though so called laboratory shift was not avoidable. ${ }^{6}$

Only a few articles have appeared to discuss the variability of the HDL-C concentration among healthy subjects. Laskarzewski et al tracked the concentrations of lipid and lipoprotein (including HDL-C) in the blood of 108 children and found that initial and subsequent HDL-C concentrations were highly correlated with $r=0.60$ and 0.53 for observations two and three years apart. ${ }^{7} \mathrm{Mj} ø \mathrm{~s}$ et al followed serum concentrations of HDL-C, TC, and others at 12-18 week intervals for 60 weeks in 17 male and 11 female young adults and observed that the mean within subject CV was smallest with TC $(6 \cdot 7-7 \cdot 8 \%)$, followed by HDL-C (8.4-9.4\%). ${ }^{8}$ The present observation-that is, very high interyear correlations ( $r>0.60$ for both HDL-C and TC) and small within subject CVs $(10.9 \%$ for total 170 women and $8.3 \%$ for 31 selected women as CV for HDL-C; $10.1 \%$ for both the former and the latter as CV for TC-is in good agreement with these reports.

It is interesting to note the association in the variation of $\mathrm{HDL}-\mathrm{C}$ and TG. The within subject CVs of both HDL-C and TG were larger in the non-normolipidaemic group than in the normolipidaemic group, and the increases were even more remarkable when the premenopausal women at the ages of 40-59 were studied to exclude confounding factors for serum lipids such as age and menopause. ${ }^{1}$ Inverse relation of HDL-C concentrations with TG concentrations among healthy subjects had been also observed, ${ }^{13} 8$ which led Mjøs et al to consider that the fluctuation of HDL-C with time is related in part to variation in TG-rich lipoprotein metabolism ${ }^{\mathbf{8}}$; the role of HDL-C in lipoprotein metabolism has been discussed by
Havel ${ }^{9}$ and Eisenberg. ${ }^{10}$ Thus HDL-C concentration of a given examinee would be best evaluated in the health examination when TG and other lipid related items are measured concurrently; as the results of the present study suggest, the HDL-C concentration of the given examinee will not be physiological even if the value stays within the normal range set by the laboratory concerned, when the TG concentration is raised.

Requests for reprints to: Professor M Ikeda.

\section{References}

${ }^{1}$ Chiba K, Koizumi A, Kumai M, Watanabe T, Ikeda M. Nationwide survey of high-density lipoprotein cholesterol among farmers in Japan. Prev Med 1983; 12: 508-22.

${ }^{2}$ Albrink MJ, Krauss RM, Lindgren FT, Groeben J Von der, Wood PD. Intercorrelations among plasma high density lipoprotein, obesity and triglycerides in a normal population. Lipids 1980; 15: 668-76.

3 Jacobs DR, Hunninghake DB, Dempsey ME, et al. Blood lipids and lipoproteins in a Minnesota urban population. J Chronic Dis 1980; 33: 395-406.

${ }^{4}$ Gordon T, Castelli WP, Hjortland MC, Kannel WB, Dawber TR. High density lipoprotein as a protective factor against coronary heart disease. Am J Med 1977; 62: 707-14.

${ }^{5}$ Miller NE, Førde OH, Thelle DS, Mjøs OD. The Thoms heart study-high density lipoprotein and coronary heart disease: a prospective case-control study. Lancet 1977; i: 965-7.

${ }^{6}$ Cresanta JL, Srinivasan SR, Foster TA, Webber LS, Berenson GS. Distributions of serum lipoproteins in children by repeated measurements. Prev Med 1983; 12: 554-68.

${ }^{7}$ Laskarzewski P, Morrison JA, de Groot L, et al. Lipid and lipoprotein tracking in 108 children over a four-year period. Pediatrics 1979; 64: 584-91.

${ }^{8}$ Mjøs OD, Bjøru SN, Henden T, Thelle DS, Førde OH, Miller NE. A longitudinal study of the biological variability of plasma lipoproteins in healthy young adults. Atherosclerosis 1979; 34: 75-81.

${ }^{9}$ Havel RJ. Lipoprotein biosynthesis and metabolism. Ann NY Acad Sci 1980; 348: 16-29.

${ }^{10}$ Eisenberg S. Plasma lipoprotein conversions: the origin of low-density and high-density lipoproteins. Ann NY Acad Sci 1980; 348: 30-47. 